\title{
VIDEO JUEGOS: ADICCIÓN Y FACTORES PREDICTORES
}

\section{Video Games: Addiction and predictors}

\author{
Miguel Vallejos y Walter Capa*
}

\begin{abstract}
Resumen
Se evalúa el modo cómo están relacionados la funcionalidad familiar y los estilos interactivos (toma de decisiones y tendencia al riesgo) con la adicción a los video juegos en los adolescentes, así como las consecuencias que genera la adicción a los juegos en la asertividad, agresividad y rendimiento académico. Se empleó una muestra probabilística estratificada de escolares de centros educativos estatales de secundaria de Lima Metropolitana, incluyendo escolares de ambos sexos con edades entre 11 y 18 años. Los resultados indican que el 85\% de los adolescentes acceden a los videojuegos, aun cuando los varones juegan más que las mujeres, tanto las mujeres (70.6\%) como los hombres (94.7\%) hacen uso alto del videojuego. Destacan entre los lugares donde se juega los videojuegos la propia casa (40\%) y en las cabinas de Internet (42\%). En cuanto a la relación entre el uso de videojuegos y las variables psicológicas (funcionamiento familiar, toma de decisiones y comportamiento agresivo) encontramos la variabilidad explicada del modelo es de 14\%, siendo el factor explicativo más importante la funcionalidad familiar $(\beta=-0.256, p<0.01)$. Asimismo encontramos que cuanto menor es la asertividad y mayor la agresividad entonces el rendimiento académico es bajo, relaciones que están condicionadas por el mayor acceso o uso de los videojuegos.
\end{abstract}

Palabras clave: Adicción, asertividad, agresividad, adolescentes, toma de decisiones, funcionalidad familiar, rendimiento académico, videos juegos.

\begin{abstract}
Assesses the way relate the familiar and interactive styles (decision-making and tendency to risk) with the video game addiction among adolescents, as well as the consequences that generates addiction games assertiveness, aggressiveness and academic performance. It employed a stratified probability sample of schoolchildren in state secondary schools Metropolitan Lima, including schoolchildren of both sexes aged between 11 and 18 years. The results indicate that $85 \%$ of adolescents have access to video games, even if the boys play more than women, while women (70.6\%) and men (94.7\%) make use of high videogame. Notable among the places where the games are played the home (40\%) and in the booths Internet (42\%). As to the relationship between the use of video games and psychological variables (family functioning, decision making and aggressive behavior) are explained variability of the model is $14 \%$, being the most important factor explaining the functionality family $(\beta=-0.256$, $p<0.01)$. We also found that the lower the greater assertiveness and aggressiveness then academic performance is low, relationships that are conditioned by the increased access or use of the games.
\end{abstract}

Key works: Addiction, assertiveness, aggressiveness, adolescents, interactive styles (decision-making and tendency to risk), family functioning, academic performance, video games.

* Facultad de Psicología, Universidad Nacional Federico Villarreal, Perú. 


\section{INTRODUCCIÓN}

En la vida diaria están presentes la televisión, los videojuegos, la música y otros medios audiovisuales. La investigación ha corroborado los efectos notables e insidiosos de estos medios sobre los niños, manifestados cotidianamente por comportamientos agresivos, miedos y desensibilización a la violencia (Sánchez, Reyes \& Hernández, 2000). Concluyen estos investigadores que durante los videojuegos los niños quedan expuestos a caracterizaciones ficticias en que la violencia se representa de manera poco realista (cuando está caracterizada sin ninguna consecuencia negativa).

En Sudamérica nuestro país ocupaba el cuarto lugar más alto en concurrencia de usuarios a cabinas de Internet (2,500,000 usuarios), del total de personas que hacían uso del Internet $65 \%$ tenía edades comprendidas entre 12 y 24 años (Apoyo, Opinión y Mercado, 2002) y el $75 \%$ de servicio de internet era ofrecido por cabinas públicas (Moran, 2004). Si bien el Internet tiene diversos usos educativos e informativos, sin embargo se ha constituido en una fuente de riesgo, así por ejemplo la accesibilidad a los videojuegos en red en todos los sectores socioeconómicos está propiciando la aparición conductas adictivas en los adolescentes, problema que puede llegar a constituirse en una seria amenaza social. Así por ejemplo España es el país con el mayor número de jugadores patológicos en toda Europa (Secades y Villa, 1998).

Es importante precisar que no sólo es la accesibilidad a los juegos en red lo que ocasiona la aparición de conductas adictivas en los adolescentes, sino que como refiere Estalló (1994) existen factores (psicológicos, sociales, legales, familiares y de ocio) condicionantes de la conducta adictiva.

En relación a los videos juegos Crawford (1984), referido por Estallo J. (1997), establece una clasificación en dos grandes grupos, juegos de habilidad y de acción por un lado y juegos de estrategia por otro. Los del primer grupo serían aquellos videojuegos que implicarían el uso de habilidades visomotoras, en tanto que los del segundo grupo incluirían un amplio abanico de aptitudes, que podríamos resumir en: Estrategias de solución de problemas, establecimiento de relaciones causales y toma de decisiones.
De acuerdo con Buchman y Funk (1996), referido por Mendiz, A.; Pindado, J.; Ruiz, J. y Pulido, J. (2001), los videojuegos han pasado a ser la principal causa de pérdida de tiempo entre los adolescentes.

\section{ADICCIÓN A LOS VIDEOS JUEGOS:}

Hasta hace algún tiempo, la concepción de la adicción estaba circunscrita a las sustancias químicas que producían dependencia, y no se había determinado ni sistematizado aún la clínica de las adicciones atípicas o no convencionales; en ese unívoco enfoque el factor de riesgo era definido entonces como un elemento de naturaleza individual o social cuya presencia pone en riesgo a un individuo de que use drogas (Echeburua, 1999). Actualmente se comprende a las adicciones con una concepción mayor, no tan sólo limitándose a las sustancias psicoactivas, sino todo relación que tiene el individuo, ya sea de carácter químico o psicológico, en cuya interacción presenta una obsesión y pérdida del autocontrol con consecuencias negativas y que a pesar de ello vuelve a entrar en relación sin importarle su estabilidad y de los demás. Para una mejor comprensión se puede entender a la adicción a decir de algunos autores como: "una enfermedad auto y heterodestructiva, crónica y progresiva, de curso variable mediante un vínculo patológico (conducta de descontrol) que establece un individuo con: una sustancia que causa neuroadaptación, un aparato o instrumento (generalmente electrónico), una actividad físico mental o una persona, causándole trastornos en su bioquímica, fisiología, sistema de comportamiento cognitivo-emocional y entorno socio-familiar" (Vacca, 1998; p. 47).

Esto viene a indicar que el individuo se ve llevado por su adicción y que cuando no puede llevarla a la práctica o realizarlo se encuentra mal. Como consecuencia de esto, la adicción se convierte en centro de su vida, o en una parte muy importante dentro de ella, descuidando el resto de sus facetas, tanto profesionales como personales. Su incapacidad de controlar la conducta le hace sentirse mal, cayendo paulatinamente en un estado de deterioro y en un círculo vicioso ya que, aun queriendo salir de su adicción, le va a ser muy difícil, si no imposible, conseguirlo por sí mismo. 


\section{Funcionalidad familiar:}

La familia es la principal responsable del cuidado y la protección de los niños desde la infancia a la adolescencia. La introducción de los niños a la cultura, los valores y las normas de la sociedad se inicia en la familia. Para que su personalidad se desarrolle plena y armónicamente, los niños deben crecer en un ambiente familiar y en una atmósfera de alegría, amor y comprensión (UNICEF, 1991). Estos productos y constructos sociales son susceptibles de ser transformados. No pueden ser tenidos por figuras inmutables e indiscutibles por cuanto derivan de procesos de producción social y de los mecanismos sociales en que éstos se apoyan.

La niñez se ubica dentro del juego de esas coordenadas múltiples de orden económico, social y político. Es a partir de este dato que hay que buscar la relación existente entre la niñez y las pautas, prácticas y creencias de crianza (Andolfi, 1985).

\section{Estilos Interactivos (personalidad):}

Este constructo de acuerdo con Ribes y Sánchez (1990; citados por Ribes, 1990) se llaman estilos interactivos -personalidad-. Dado que este término psicológico no está referido a una estructura interna que causa conductas, sino como modos particulares de actuar frente a situaciones. Así, los estilos interactivos, implican la manera idiosincrásica y consistente en que una persona se enfrenta con una situación dada. Los estilos, modulan la velocidad, la oportunidad y la precisión de las conductas requeridas para una situación que demanda eficacia.

\section{Asertividad:}

Se conceptúa como un factor disposicional (Capa, 1997 y 1998) que probabiliza la ocurrencia de conductas asertivas. La conducta asertiva se define por el criterio de efectividad en las contingencias de interacción social de modo que "resuelvan un problema o produzcan resultados de consecuencias deseables o requeridas..." (Capa, 1998). Desde esta concepción las morfologías reactivas del individuo, ampliamente operativizadas en la literatura conductista como: saber expresar reclamos, rechazos, derechos, hacer o decir lo que es correcto, entre otros, son inclusivas a la categoría funcional de la conducta asertiva.

\section{Agresividad:}

Bandura afirma que el componente principal y lo que podría estar determinando la característica del comportamiento agresivo y violento es un componente de interacción con el ambiente, es decir, un proceso de aprendizaje llamado imitación.Bajo esta misma línea el término agresión en un sentido psicológico ha sido conceptuado como una clase de respuesta psicosocial emitida ante estímulos sociales aversivos y frustrantes (Anicama, 1996); esto implica un conjunto de respuestas componentes y un conjunto de eventos estímulos en interacción, actuando a diferente nivel de expresión del organismo.

\section{Rendimiento Académico:}

En cuanto a los factores asociados negativamente al rendimiento académico, Patterson y cols (1975; véase Michelson et al., 1987) sostienen que es bastante habitual que los niños socialmente negativos y agresivos presenten dificultades escolares, obteniendo unos niveles más bajos que sus compañeros de clase. De manera similar Michelson et al. (1987) señalan que los niños agresivos tienden a adquirir las materias académicas en una menor proporción en comparación con los niños no agresivos y que además de ser menos populares entre sus compañeros, también manifiestan una mayor incidencia de fracaso escolar. Kohn y Rosman (1972; véase Michelson et al, 1987) investigaron la relación entre el funcionamiento social y emocional preescolar, así mismo su posterior rendimiento escolar y éxito intelectual. Los comportamientos asertivos de estos niños preescolares fueron asociados con una buena disposición escolar y el éxito intelectual en los años posteriores. Señalan los autores, que los resultados "sugieren que el niño que es curioso, despierto y asertivo aprenderá más de su medio ambiente, y el niño que es pasivo, apático y retraído aprenderá menos, dado su contacto disminuido". Del estudio reportado por Ghezzi y Bijou (1994).

La presente investigación busca desde una perspectiva psicológica ofrecer un modelo explicativo de la adicción a los videos juegos en la población adolescente, en términos de los factores predisponentes o moduladores de dicha problemática (funcionalidad familiar y estilos interactivos) así como los efectos que acarrea en el plano psicológico (asertividad y agresividad) y escolar (rendimiento académico). 
En el contexto de lo descrito en la presente investigación nuestros problemas a resolver son: ¿Cómo están relacionados la funcionalidad familiar y los estilos interactivos (toma de decisiones y tendencia al riesgo) con la adicción a los videos juegos en los adolescentes? y ¿qué consecuencias genera la adicción a los juegos en la asertividad, agresividad y rendimiento académico?

\section{MÉTODO}

\section{Población y Muestra:}

La población lo constituyeron 4954 adolescentes que se encontraban cursando entre primero y quinto año de secundaría en 6 colegios estatales de un distrito de Lima Metropolitana. La mencionada población incluyó a adolescentes de ambos sexos, con edades entre los 11 y 18 años cumplidos al momento de la evaluación.

Para el presente estudio se estimó una muestra probabilística de tipo estratificado por colegio, para ello se asumirán como estimadores un nivel de confianza de $95 \%$ ( $\mathrm{z}=1.96$ ), error de $2 \%$ y probabilidad de ocurrencia $50 \%(\mathrm{p}=50 \%$ y $\mathrm{q}=50 \%)$.

\section{Diseño y Variables:}

La investigación correspondiente es de diseño noexperimental o ex-post-facto, de tipo correlacional (Alarcón, 1991; Hernández, Fernández y Baptista, 1998; Kerlinger, 1994).

Siendo desde el punto de vista de la naturaleza relacional entre las variables una investigación multivariante (Kerlinger y Lee, 2002; Polit \& Hungler, 1997).

Las variables de estudio son:

\section{Instrumento y Materiales:}

Se utilizó una Ficha Socio-demográfica, instrumento que nos permitió recabar los datos socio-demográficos, tipo de familia e información referente al rendimiento académico; la Escala para problemas de adicción a videojuegos (Problem video game playingPVP), construida por Tejeiro y Moran (2002) con el objetivo de validar una escala que midiese el problema de adicción a los videojuegos. Resultados hallados muestran que el PVP es unidimensional y tiene una consistencia aceptable (alfa de Cronbach $=0.69$ ). El patrón de asociación entre las puntuaciones de las escalas y las medidas alternativas de los jugadores problema apoyan la validez de constructo (elevados puntajes correlacionan con elevada frecuencia de juego, más larga duración de la sesión, percepción de los padres del problema y puntuación en la gravedad de la escala de dependencia); Cuestionario de Personalidad (estilos interactivos):

La Lista de Chequeo Conductual de estilos Interactivos ha sido diseñado por Montes de Oca (1995) y ha sido validado mediante los criterios de jueces y de constructo (Inga y Montes de Oca, 1999). Este instrumento permite identificar siete estilos siguientes: Toma de decisiones, tendencia al riesgo, tolerancia a la frustración, nivel de logro, tendencia a la transgresión, impulsividad- no impulsividad. En la presente investigación se tomaron los estilos de toma de decisiones y tendencia al riesgo; Escala de Asertividad: Para la investigación se construyó un autoreporte con opciones de respuesta tipo likert con el objetivo de evaluar la asertividad. Este instrumento consta de 16 items. Sus propiedades métricas se reportan en el estudio y el Cuestionario de Agresión- AQ: Este autoreporte denominado Cuestionario de Agresión (Aggression Questionnaire - AQ) fue construido por Buss y Perry (1992), traducido y adaptado al español por Andreu, Peña y Graña (2006) para población de jóvenes y adolescentes madrileños. La fiabilidad de las cuatro subescalas que componen el Cuestionario de Agresión fueron diferencialmente satisfactorias. Respecto a la validez de constructo presentada por el instrumento, el análisis factorial confirmatorio, realizado a través de un modelo tetradimensional de ecuaciones estructurales, confirmó que, en términos generales, este cuestionario permite medir de forma válida. Para su uso en nuestra investigación se estimaron sus propiedades psicométricas correspondientes.

\section{RESULTADOS}

En la Tabla 1 se observa $86.1 \%$ ( 8 de cada 10 ) de los adolescentes hacen uso de los video juegos. Sólo un $13.9 \%$ aún no ha jugado. 
Tabla 1

Prevalencia de uso de videojuegos en los adolescentes

\begin{tabular}{lcc}
\hline $\begin{array}{l}\text { Uso de } \\
\text { videojuegos }\end{array}$ & Frecuencia & Porcentaje \\
\hline No & 82 & 13,9 \\
$\mathrm{Si}$ & 509 & 86,1 \\
Total & 591 & 100,0 \\
\hline
\end{tabular}

Apreciamos en la Tabla 2 que $94.7 \%$ de los varones ha jugado a los videojuegos, en tanto que, en el grupo de mujeres $70.6 \%$ ha hecho uso de estos juegos.

Tabla 2

Prevalencia de uso de videojuegos en los adolescentes según sexo

\begin{tabular}{lrrrr}
\hline & \multicolumn{2}{c}{ Mujeres } & \multicolumn{2}{c}{ Varones } \\
$\begin{array}{l}\text { Uso de } \\
\text { videojuegos }\end{array}$ & Frecuencia & Porcentaje & Frecuencia & Porcentaje \\
\hline No & 62 & 29.4 & 20 & 5.3 \\
Si & 149 & 70.6 & 355 & 94.7 \\
Total & 211 & 100,0 & 375 & 100.0 \\
\hline
\end{tabular}

La Tabla 3 indica que $13.6 \%$ de los adolescentes juegan diariamente a los video juegos y $22.3 \%$ de manera interdiaria. Asimismo se observa que los que sólo juegan los fines de semana representa a $42.7 \%$ de los adolescentes.

Tabla 3

Análisis porcentual del número de veces jugados a la semana

\begin{tabular}{lccc}
\hline $\begin{array}{l}\mathbf{N}^{\mathbf{o}} \text { veces jugados } \\
\text { al semana }\end{array}$ & Frecuencia & Porcentaje & $\begin{array}{c}\text { Porcentaje } \\
\text { acumulado }\end{array}$ \\
\hline Fines de semana & 226 & 42.7 & 42.7 \\
Dos veces por semana & 113 & 21.4 & 64.1 \\
Interdiario & 118 & 22.3 & 86.4 \\
Diario & 72 & 13.6 & 100.0 \\
Total & 529 & 100.0 & 100. \\
\hline
\end{tabular}

En la Tabla 4 se observa 4 de cada 10 adolescentes hace uso de los video juegos en sus hogares, en tanto que otro similar (42.1\%) accede a dichos juegos en las cabinas de internet.
Tabla 4

Análisis porcentual de los lugares donde se accede a los videojuegos

\begin{tabular}{lccc}
\hline $\begin{array}{l}\text { Lugares donde } \\
\text { se accede }\end{array}$ & $\begin{array}{c}\text { Frecuencia } \\
\text { acumulado }\end{array}$ & Porcentaje & Porcentaje \\
\hline Casa propia & 209 & 40,0 & 40,0 \\
Casa de amigos/as & 40 & 7,6 & 47,6 \\
Escuela & 40 & 7,6 & 55,3 \\
Cabinas de internet & 220 & 42,1 & 97,3 \\
Otros lugares & 14 & 2,7 & 100,0 \\
Total & 523 & 100,0 & \\
\hline
\end{tabular}

La Tabla 5 muestra que 7 de cada 10 adolescentes hace de los videos juegos entre 1 a 5 horas a la semana, y aproximadamente 2 de cada 10 entre 6 a 10 horas semanales. De 20 a más horas contiene a $6 \%$ de los niños jugares a videos.

Tabla 5

Análisis porcentual de cantidad de horas jugadas a la semana

\begin{tabular}{lccc}
\hline $\begin{array}{l}\text { Cantidad de } \\
\text { horas jugadas }\end{array}$ & $\begin{array}{l}\text { Frecuencia } \\
\text { acumulado }\end{array}$ & Porcentaje & Porcentaje \\
\hline 1 a 5 horas & 372 & 70.1 & 70.1 \\
6 a 10 horas & 96 & 18.1 & 88.1 \\
11 a 20 horas & 31 & 5.8 & 94.0 \\
20 a 30 horas & 10 & 1.9 & 95.9 \\
Más de 30 horas & 22 & 4.1 & 100.0 \\
Total & 531 & 100,0 & \\
\hline
\end{tabular}

La Tabla 6 muestra que 2 de cada 10 adolescentes presenta dependencia a los videos juegos, y 7 de cada 10 adolecentes abusa de los videojuegos y por último se observa que un 25.3 por ciento hace uso de los videojuegos.

Tabla 6

Análisis porcentual de los tipos de jugadores de videojuegos

\begin{tabular}{lccc}
\hline Tipo de Jugador & Frecuencia & Porcentaje & $\begin{array}{l}\text { Porcentaje } \\
\text { acumulado }\end{array}$ \\
\hline Usador & 150 & 25.3 & 25.3 \\
Abusador & 311 & 52.5 & 77.9 \\
Dependiente & 131 & 22.1 & 100.0 \\
Total & 592 & 100.0 & 100.0 \\
\hline
\end{tabular}


La Tabla 7 nos indica que el modelo propuesto para explicar las probabilidades de adicción a los videojuegos es altamente significativo $(\mathrm{F}=23.2$, $\mathrm{p}<0.01$ ). Observándose que la variabilidad explicada del modelo es de 14\%; asimismo las relaciones entre las variables independientes de la ecuación y el uso de los videojuegos (variable dependiente) todas son significativas. Siendo el factor explicativo más importante la funcionalidad familiar $(\beta=-0.256$, $\mathrm{p}<0.01)$.

Tabla 7

Análisis de Regresión Múltiple para el modelo explicativo de adicción a los video juegos

\begin{tabular}{|c|c|c|c|c|}
\hline Regresión Múltiple & \multicolumn{3}{|l|}{0.370} & \\
\hline $\mathrm{R} 2$ & \multicolumn{3}{|l|}{0.137} & \\
\hline $\mathrm{F}$ & \multicolumn{2}{|l|}{23.201} & Signif. F & 0.000 \\
\hline \multicolumn{5}{|c|}{ Variables Indep. en la ecuación } \\
\hline & & Beta & $\mathbf{t}$ & $\mathbf{p}$ \\
\hline \multicolumn{2}{|c|}{ Funcionalidad familiar } &,- 256 & $-6,423$ & ,000 \\
\hline \multicolumn{2}{|l|}{ Toma de decisiones } &,- 111 & $-2,820$ & ,005 \\
\hline \multicolumn{2}{|l|}{ Agresividad } & , 136 & 3,457 & ,001 \\
\hline \multicolumn{2}{|c|}{ Rendimiento académico } & -.082 & -2.096 & ,036 \\
\hline
\end{tabular}

La Tabla 8 nos indica que el modelo formulado para predecir el nivel de rendimiento académico es altamente significativo $(\mathrm{F}=9.47, \mathrm{p}<0.01)$. La variabilidad explicada del rendimiento académico a partir de las variables independientes es de 6\%. Los pesos beta estandarizado nos indica que, aun cuando todos los factores son significativos en explicar la variabilidad del rendimiento académico, sin embargo el factor de mayor importancia en la predicción del rendimiento académico viene a ser la toma de decisiones $(\beta=0.117, \mathrm{p}<0.01)$. Observándose que cuanto más apropiados o pertinentes son las decisiones que toman los adolescentes mayor es su rendimiento académico, en contraste las decisiones incorrectas conllevan al bajo rendimiento académico.
Tabla 8

Análisis de Regresión Múltiple para el modelo predictor del rendimiento académico

\begin{tabular}{|c|c|c|c|}
\hline Regresión Múltiple & 0.246 & & \\
\hline $\mathrm{R}^{2}$ & 0.061 & & \\
\hline $\mathrm{F}$ & 9.472 & Signif. F & 0.000 \\
\hline \multicolumn{4}{|c|}{ Variables Independientes en la ecuación } \\
\hline & Beta & $\mathbf{t}$ & $\mathbf{p}$ \\
\hline Video juego &,- 094 & $-2,264$ &, 024 \\
\hline Asertividad &, 101 & 2,503 & 013 \\
\hline Agresividad &,- 124 & $-3,041$ &, 002 \\
\hline Toma de decisiones & .117 & 2.871 & ,004 \\
\hline
\end{tabular}

\section{DISCUSIÓN}

Disertar acerca de adicción a los videojuegos resulta polémico, como refieren los estudios, el rápido crecimiento en popularidad de los videojuegos, junto con la avidez con la que juegan los jóvenes, ha generado inquietud entre los científicos sociales, padres de familia y políticos en cuanto al efecto que estos juegos tienen en los usuarios (Martínez, Fernández-Alba y Salgado, 2004; Tejeiros, 2001). A decir de los hallazgos de la presente investigación, minimizar podría llevarnos a negar esta realidad y adoptar una ceguera social respecto a un problema emergente que esta involucrando a niños y adolescentes, generándoles consecuencias negativas por su práctica no controlada.

De acuerdo a nuestros datos el 85 por ciento de la muestra estudiada reporta practicar los videojuegos, siendo los varones quienes juegan más que las mujeres. Respecto a los días de juego encontramos que más del cincuenta por ciento juega más de dos por semana. Estos datos se acercan a los hallazgos reportados en España y Chile (Martínez, Fernández-Alba y Salgado, 2004; Rodríguez, Mejias, Calvo, Sánchez y Navarro, 2002; Sommer, 2006). Para Sommer (2006) esta frecuencia de hacer uso de los video juegos en los niños por más de una hora diaria, entre lunes y viernes, conlleva cinco veces más probabilidades de caer en el grupo de alto riesgo.

Respecto a las diferencias de uso en relación al género, nuestros hallazgos declaran que los adolescentes de nuestro medio usan más los videojuegos en comparación a otras latitudes; nuestros hallazgos evidencian que la mayoría de los adolescentes tanto las muje- 
res como los hombres hacen uso del videojuego, siendo las mujeres un 70,6\% y un $94,7 \%$ los varones. Estos resultados, se encuentran muy cercanos a los datos reportados en muestras españolas donde el $72,9 \%$ de varones y 43,4\% de mujeres declaraban usar el video juegos (Mejia et al., 2000) y muy lejos de nuestro vecino país del sur, en Chile se halló que el $48 \%$ de la muestra estudiada calificaba dentro del grupo de alto riesgo y el 35\% de las mujeres no cumplía ningún criterio, es decir, simplemente no jugaban y en los varones sólo el $9 \%$ declaraban no haber usado videojuegos. Asimismo Sommer (2006) reporta que el 65\% de las mujeres se incluían en el grupo de bajo riesgo, mientras que el $70 \%$ de los hombres lo hicieron en el grupo de alto riesgo. Para Mejias (2002) el uso de videojuegos es un comportamiento cultural claramente masculino (los varones juegan el doble). Esto hace que el mercado se organice para los varones, y así se refuerza la tendencia de género.

Un aspecto importante que hay que tener en cuenta, es el lugar donde se juega los videojuegos, al observar los resultados donde se juega encontramos que es en la propia casa (40\%) y en las cabinas de Internet (42.1\%), datos que indican que el hogar se ha convertido en lugar de riesgo para mantener este comportamiento y que el control parental debe ser clave en este aspecto. Como sostiene Sommer (2006) en este punto es fundamental la supervisión de los padres, ya que aquellos menores cuyos padres no controlaban la cantidad de horas al día que sus hijos dedicaban al juego presentaban más riesgo de desarrollar adicción.

Por otro lado, cuando se analiza el uso problemático del videojuego y sus relación con otras variables predictoras, hallamos que existe una correlación moderada entre el uso del videojuegos y las variables relacionadas como el funcionamiento familiar, toma de decisiones, comportamiento agresivo y rendimiento académico, donde podemos observar que hay una relación inversa entre funcionamiento familiar y el uso del videojuegos, es decir, a menor funcionamiento familiar mayor uso del videojuego; igualmente con la toma de decisiones y el rendimiento académico, concluyéndose que a menor toma de decisiones apropiadas mayor uso de los videojuegos, y por último si hay mayor uso de los videojuegos hay problemas en el rendimiento académico. Estos hallazgos coinciden con los reportados en otros estudios, en Chile los niños que pertene- cen a familias disfuncionales presentan el doble de riesgo que aquellos que están cómodos, son escuchados, queridos y apoyados; respecto al funcionamiento escolar, también se encontró asociación entre los niños que dedicaban mayor tiempo a los videojuegos con un menor rendimiento escolar (Sommer, 2000). En España los estudios reportan que casi el $60 \%$ de los jugadores nunca generaron problemas por jugar y pero si el $40 \%$ de los jugadores reportaron problemas escolares y familiares, alteraciones del ritmo del sueño y abandono de alguna obligación, así como discutir con los amigos y los padres, no hacer las tareas, dejar ir a clases, siendo estos problemas mucho más frecuentes en los varones (Espinosa, 2000; Mejia et al., 2002).

Ante estos hallazgos consideramos importante sugerir a los padres de familia, educadores y a la sociedad en general que durante los días hábiles de la semana los niños dediquen menos de una hora diaria a los videojuegos y que durante el fin de semana el tiempo sea controlado y supervisado, estableciendo límites y favoreciendo otras actividades. Así mismo tener presente, el punto fundamental, la supervisión parental, ya que aquellos menores cuyos padres no controlaban la cantidad de horas al día que sus hijos dedicaban al juego tienen más riesgo de desarrollar adicción.

\section{REFERENCIAS}

Andolfi, M.; Zwerling, I. (1985). Dimensiones de la terapia familiar. Barcelona: Paidós.

Andreu, J.M.; Peña, M.E. \& Graña, J.L. (2002). Adaptación psicometrica de la versión española del Cuestionario de Agresión. Psicothema,14(2): 476-482

Anicama, J. (1996). Efectos de un programa ART de sustitución y prevención de la violencia en menores de alto riesgo. Revista Psicología Contemporánea, 3(2):12-21.

Bandura, A. (1973). Agresión: un análisis de aprendizaje social. Englewood Cliffs: Prentice Hall.

Becoña, E. \& Vásquez, F. (2001). Heroína, cocaína y drogas de síntesis. Madrid: Síntesis.

Buss, A.H. y Perry, M. (1992). The aggression questionnaire. Journal of Personality and Social Psychology, 63, 452-459.

Cabello, A.M. \& Hernández, L. (1997). El padre en la crianza y el desarrollo infantil. Santiago de Chile: Fundación Bernard van Leer

Cabello, A.M.; Ochoa, J. y Filp, J. (1992). Pautas y 
prácticas de crianza de niños y niñas entre 0 y 6 años de sectores pobres. Santiago de Chile: CELAM UNICEF

Capa, W. (1997). Relación entre conducta asertiva y rendimiento escolar en estudiantes de secundaria de nivel socioeconómico medio-alto. Tesis de Licenciatura en Psicología. Lima: UNFV.

Chadwick, C. (1979). Teorías del aprendizaje. Santiago: Tecla.

Cortes, A.; Romero, P. \& Flores, G. (2006). Diseño y validación inicial de un instrumento para evaluar prácticas de crianza en la infancia. Universitas Psychologica de Bogotá, 5 (1): 37-49.

Echeburúa, E. (1999). ¿Adicciones sin drogas? Bilbao: Desclée de Brouwer.

Escobar, X. y Olmedo, A. (1993). Estudio exploratorio descriptivo de las características de la figura paterna en una muestra de escolares de 8 a 11 años de edad con padre ausente. Memoria para Optar al Título de Psicólogo. Santiago: Universidad de Chile.

Espinosa, D. (2000) Tolerancia en los videojuegos:una lectura con mapa axiologicos. Tesis de Doctorado. Universidad de Alicante. Biblioteca Virtual Miguel De Cervantes. Alicante.

Estallo, J. (1994). Videojuego, personalidad y conducta. Psicothema, 6 (2), 181-190

Estallo, J. (1997). Psicopatología y videojuegos. Recuperado el 20 de julio de 2010 de: http:// www.ub.edu/personal/videoju.htm

Ghezzi, P. y Bijou, S. (1994). El entrenamiento en habilidades sociales en niños solitarios medianamente retardados. En L. Hayes, E. Ribes y F. López (Eds), Psicología interconductual: Contribuciones en honor a J. R. Kantor. (pp 91-110). Guadalajara, México: Universidad de Guadalajara.

Kantor, J. R. (1978). Psicología interconductual. México: Trillas.

Martínez, Cepeda, Fuentes y Burgos (1995) Evaluación del Aprendizaje. En Rueda, M (Ed) Procesos de enseñanza y aprendizaje I. México: Consejo Mexicano de Investigación Educativa.

Martínez, R; Fernández-Alba, A y Salgado, A. (2004). Patrones de ocio en los adolescentes de la Comunidad de Madrid. Conductas de riesgo: de Tomb Raider al botellón. Madrid.

Mendiz, Alfonso; Pindado, Julián; Ruiz, Javier; Pulido, José (2001). Videojuegos y educación, una revisión crítica de la investigación y la reflexión sobre la materia. En:
Videojuegos y educación. Ministerio de Educación y Ciencia de España. Recuparado el 20 de agosto de 2010.

Michelson, L; Sugai, D; Wood, R y Kazdin, A. (1987). Las habilidades sociales en la infancia: Evaluación y tratamiento. Barcelona: Martínez Roca.

Moran, J. (2004). El servicio de Internet en la biblioteca del Instituto Cultural Peruano Norteamericano de San Miguel. Tesis de Licenciatura en Bibliotecología y Ciencias de la información. Lima: UNMSM.

Orellana, O. y García, L. (1996). Violencia y representaciones sociales en escolares. Revista Peruana de Psicología, 1(1): 26-39.

Papalia, D. E. \& Wendkos, S. (1992). Desarrollo Humano. Bogotá, Colombia: Mc Graw Hill

Ribes, E. y López, F. (1985). Teoría de la conducta, un análisis de campo y paramétrico. México: Trillas.

Rodríguez, E; Mejias, I; Calvo, A; Sánchez, E. y Navarro, J. (2002). Jóvenes y Videojuegos: Espacios, Significación y Conflictos. Fundación de Ayuda a la Drogodependencias (FAD) e INJUVE. Madrid

Rueda, M (1995). Procesos de Enseñanza y Aprendizaje I. México: Consejo Mexicano de Investigación Educativa.

Sánchez, N.; Reyes, U. \& Hernández, M. (2000). Videojuegos, reporte preliminar. Revista mexicana de puericultura y pediatría, 7(41): 150-156.

Secades, R \& Villa, A. (1999). El Juego patológico, prevención y tratamiento en adolescentes. Psicothema, 11(3), 702-703

Sommer, K (2006) Niños chilenos en las redes de los videojuegos. Primer estudio nacional sobre abuso del entretenimiento digital. Recuperado el 8 de agosto de 2010 de: http://www.lanacion.cl/prontus_noticias/site/ artic/20061017/pags/20061017212113.html

Tejeiro, R \& Bersabé, R Morán (2002). Universidad de Málaga, Málaga, Spain2 Measuring problem video game playing in adolescents. Addiction, 97 (2), 16011606, December 2002

Tejeiro, R (2001) La adicción a los videojuegos: Una revisión. Adicciones, 3 (4), 407 - 413.

Tejeiro, R y Pelegrina, M. (2003) Los videojuegos: qué son y cómo nos afectan. Barcelona: Ariel.

UNICEF (1991). Estado mundial de la infancia. New York: UNICEF.

Vacca, C. (1998). Los padres, los hijos y la pareja del adicto. Lima: Opción.

Fecha de recepción: 18 de junio, 2010

Fecha de aceptación: 23 de agosto, 2010 